\title{
SÍNDROME DA DOR ABDOMINAL FUNCIONAL: dor abdominal constante ou frequentemente recorrente
}

\author{
Ami D. SPERBER ${ }^{1}$, Douglas A. DROSSMAN²
}

\section{DOR ABDOMINAL CRÔNICA}

A dor abdominal é um sintoma com expressão aguda ou crônica e tem muitas etiologias. Livros e textos sobre o diagnóstico físico fornecem tabelas extensas sobre o diagnóstico diferencial da dor aguda e crônica abdominal, mas fazer um diagnóstico apenas por tentativa de encaixar os sintomas do paciente em uma lista não é suficiente. É a habilidade de escuta atenta e a integração de dados históricos em um quadro conceitual que orienta a estratégia de diagnóstico; exame físico e estudos confirmatórios são complementares ${ }^{(1)}$. Isto é particularmente verdade para a dor abdominal crônica. Na verdade, os clínicos abordam o diagnóstico e tratamento da dor aguda e crônica de forma diferente.

Realizar um diagnóstico diferencial da dor abdominal aguda parece mais compreensível, uma vez que é mais comumente gerada a partir de uma única fonte periférica. Existem características em relação à descrição dos sintomas, à localização, à evolução no tempo, e aos fatores que agravam-na e aliviam-na. Portanto, os médicos estão de acordo ao se considerar o diagnóstico de uma úlcera péptica quando o paciente descreve o início recente de dor epigástrica em queimação intermitente, que é aliviada por refeições e antiácidos e associados a fezes negras. Em contraste, é mais difícil aplicar um modelo de dor aguda em pacientes com dor abdominal crônica. Aqui, os sintomas de dor podem não se comportar da maneira esperada, sendo sempre presentes, mal localizados, associados a testes negativos de diagnóstico, e não prontamente respondem a tratamentos. As opções diagnósticas e terapêuticas deixam o médico confuso e a própria legitimidade destas condições pode entrar em questão.
O que é necessário é uma abordagem biopsicossocial mais ampla ao se aproximar da dor crônica gastrointestinal $^{(2)}$. Existem diferentes tipos de dor abdominal crônica. Muitas têm uma participação periférica, como a dor devida à pancreatite crônica ou doença inflamatória do intestino. Aqui, a natureza e a gravidade dos sintomas são atribuídas ao dano patológico subjacente. No entanto, como a dor se torna mais crônica, tratamentos direcionados para o órgão periférico tornam-se menos eficazes do que o ideal, particularmente quando há pouca ou nenhuma evidência de danos estruturais.

Quando a dor abdominal se torna uma presença constante, não se associa à uma etiologia da estrutura intestinal e é prejudicial para a vida, classifica-se como um transtorno funcional gastrointestinal. Síndrome da dor abdominal funcional (SDAF) é um distúrbio funcional gastrointestinal debilitante caracterizado pela contínua e persistente ou recorrente dor abdominal que está associada com a perda da funcionalidade diária $^{(3)}$. Não há nenhuma evidência de anormalidades estruturais (bioquímicas) para explicar o sintoma, o que não é ficção. SDAF é menos prevalente do que a síndrome do intestino irritável com a qual pode ser confundida. Devido à sua natureza debilitante, o ônus da doença em termos de absenteísmo do trabalho e utilização de serviços de saúde é substancial ${ }^{(4)}$. Embora SDAF já tenha sido classificada como um dos distúrbios intestinais funcionais, na classificação Roma III dos distúrbios funcionais gastrointestinais, foi colocada em sua própria categoria separada ${ }^{(3)}$. A dor não está relacionada com a motilidade anormal ou até mesmo com alguma sinalização visceral ${ }^{(5)} \mathrm{e}$, clinicamente, não está associada à mudança no hábito intestinal, ou alimentar, nem é aliviada pela defecação.

${ }^{1}$ Faculty of Health Sciences, Ben-Gurion University of the Negev, Beer-Sheva, Israel; ${ }^{2}$ Presidente, Rome Foundation e Codiretor, UNC Center for Functional Gastrointestinal and Motility Disorders, Division of Gastroenterology and Hepatology, University of North Carolina at Chapel Hill, Chapel Hill, New California, USA.

Correspondência: Ami D. Sperber, Faculty of Health Sciences, Ben-Gurion University of the Negev, Beer-Sheva 84105, Israel. E-mail: amy@bgu.ac.il 
A maior contribuição para a dor refere-se ao conhecimento de funcionamento anormal do cíngulo que pode estar ligado a distúrbios psicossociais ${ }^{(6-8)}$. Como existe uma escassez de literatura sobre SDAF, esta afirmação baseia-se em dados obtidos a partir de pacientes com síndrome do intestino irritável grave dolorosa e distúrbios psicológicos, o que poderia ser considerada como uma condição substituta que representa a SDAF.

Do ponto de vista fisiopatológico, a dor pode ter origens de qualquer lugar do neuroeixo. Ao ferir um dedo, a dor resulta claramente da lesão neural periférica com sinal aferente nociceptivo aumentado para o cérebro. Isto também é verdade para a inflamação aguda ou lesão visceral, tais como colecistite aguda ou obstrução intestinal. No entanto, quando um distúrbio gastrointestinal torna-se crônico, como observado anteriormente com a doença inflamatória do intestino ou a pancreatite crônica, a experiência de dor é cada vez mais influenciada pela função do sistema nervoso central. Em distúrbios gastrintestinais funcionais, os sintomas tornam-se ainda mais associados com a desregulação do centro amplificador da dor, para além da disfunção intestinal. Finalmente, em SDAF, pode haver pouca ou nenhuma perturbação gastrointestinal e o principal distúrbio é uma "percepção anormal da função intestinal normal", em vez de uma função intestinal anormal. Na verdade, o principal mecanismo de regulação da dor alterada com SDAF diz respeito à falta de inibição e, possivelmente, até mesmo à ampliação da entrada de regulamentação normal aferente pelas alterações nas centrais de controle de mecanismos de "entrada" (originários no córtex pré-frontal e cingulado e outras estruturas límbicas) ${ }^{(7)}$. Esta deficiência na inibição da dor homeostática (desinibição) pode estar relacionada com a serotonina reduzida, noradrenalina, endorfina, e atividade de outro neuropeptídeo através do circuito do sistema nervoso central medial do corno dorsal. Assim, embora a dor seja experimentada no (e atribuído ao) abdomen, estes centros cognitivos e emocionais regulam a percepção da dor, principalmente através dos mecanismos de controle de entrada do eixo cérebro - intestino. O reconhecimento do sistema nervoso central como o modulador primário da dor em SDAF é essencial para se compreender as manifestações clínicas e para o desenvolvimento de estratégias de diagnóstico e terapêuticas.

O diagnóstico da SDAF, embora baseado em sintoma, reflete o conhecimento desta relação central em vez de propriamente distúrbios intestinais.

Extensas investigações diagnósticas, na ausência de "bandeiras vermelhas" ou recursos de alarme (ver Caixa 4 na Figura 1 e Legenda 4) sugerem que tais exames são solicitados por médicos não habilitados e quando os pacientes insistem em que "algo deve ser feito" e/ou porque os profissionais temem estar "ocorrendo algo mais sério". Extensos estudos diagnósticos são injustificados, não só por não serem indicados clinicamente, mas também pelo seu alto custo e tendem a prejudicar a relação médico - paciente e a confiança terapêutica. Podem sinalizar para o paciente que o médico é inseguro quanto ao diagnóstico positivo da SDAF e reduzir a confiança geral do doente no plano de cuidados. Um objetivo importante no cuidado de pacientes com SDAF é desenvolver a compreensão dessa condição como um distúrbio biopsicossocial em que os sintomas podem ser atribuídos a disfunção do eixo cérebro - intestino. Um plano terapêutico deve ser desenvolvido e implementado com confiança e ficar clara a compreensão dos mecanismos de geração de sintomas e seu tratamento.

A maior freqüência de dificuldades psicossociais na SDAF que aumentam a experiência de dor através dessas vias centrais do sistema nervoso ${ }^{(6,9)}$ deve ser esclarecida e tratada. Esse entendimento pode levar a tratamentos comportamentais efetivos e psicofarmacológicos ${ }^{(10)}$. A base de qualquer estratégia terapêutica é a relação médico- paciente, que se baseia no reconhecimento da compaixão, do sofrimento associado com a condição dolorosa ("legitimação" de dor) e manutenção de uma postura objetiva e observadora. Outras opções terapêuticas nascem fora dessa relação básica e inclui ambas as abordagens, farmacológicas e não farmacológicas. Todos os programas de tratamento devem ser baseados em uma estratégia de múltiplos componentes, sendo o paciente como um parceiro de pleno direito. Expectativas realistas devem visar para o "cuidado" ao invés de "cura" ${ }^{(1)}$.

\section{Anamnese}

Mulher de 33 anos de idade é encaminhada ao gastroenterologista pelo médico de atenção primária (MAP) em razão de uma longa história de dor abdominal constante e grave, refratária a todos os tratamentos anteriores; associado ao problema de dor a paciente informa perda da qualidade de vida e é incapaz de trabalhar (Caixa 1, Figura 1). Não apresenta doença sistêmica conhecida que explique a dor.

O gastroenterologista obtém a história de que a paciente desenvolveu pela primeira vez episódios recorrentes de dor abdominal aos 6 anos de idade e que resultavam em ausência na escola. A frequência e a gravidade dos episódios de dor aumentaram após a menarca. Ao longo dos últimos 10 anos, a dor tem se tornado mais frequente e mais grave e nos últimos 5 anos é mais constante, ocorrendo diariamente. É descrita como surda (maçante) ou de característica espasmódica (cólica), e se localiza geralmente do meio para o abdome inferior. Especificamente, a dor não está relacionada ou é afetada pelas evacuações, pela alimentação ou pelo ciclo menstrual (Caixa 2). A paciente menciona dor intensa ao retrair e segurar o seu abdome e solicita que sejam feitos estudos diagnósticos para "encontrar e consertar" o problema (Caixa 1). Seus registros indicam que os exames físicos no passado e os estudos diagnósticos foram negativos para outros distúrbios médicos (Caixa 3). Os exames incluíram duas colonoscopias, endoscopia gastrointestinal superior, tomografia computadorizada (TC) do abdome, endoscopia por cápsula, ultrassom pélvico e exame de ressonância magnética abdominal (RM). Uma laparotomia exploratória 5 anos antes sugeriu endometriose, resultando em uma tentativa mal sucedida com uso de acetato de leuprolida. Ela também se submeteu a colecistectomia há 3 anos devido a uma baixa fração de ejeção na cintilografia utilizando DISIDA (isótopo). Não há sinais clínicos de alarme (Caixa 4). 
A doente afirma que fez mais de 30 visitas ao pronto-socorro (PS) onde geralmente recebe morfina e fenergan por via intravenosa e recebe alta com um suprimento para 1 semana de narcóticos orais, hidrocodona ou oxicodona. Na carta de encaminhamento, o seu MAP afirma que frequentemente ela precisa se reabastecer destes medicamentos, prescritos para evitar que retorne ao pronto-socorro. Foi hospitalizada 5 vezes por dor abdominal quando os tratamentos do PS não foram bem sucedidos.

Elementos detalhados adicionais de história revelam que aos 16 anos a paciente saiu de casa antes de concluir o ensino médio e após, engravidou e casou-se aos 17 anos. Após 4 anos ela abandonou o marido quando ele se tornou fisicamente abusivo. A paciente e a filha vivem atualmente com a sua mãe. Nos últimos 2 anos está incapaz para o trabalho (Caixa 1) e atualmente recebe auxílio-doença. O gastroenterologista observa que um psiquiatra consultado diagnosticou depressão maior com distúrbio de estresse pós-traumático, resultante de uma história de infância com privação familiar e abuso sexual e físico. A dor é considerada consistente com um Distúrbio de Dor Associado com Fatores Psicológicos (DSM-IV 307.80) e não há evidência de simulação de doença para fugir de responsabilidades (Caixa 7). O psiquiatra recomendou tratamento com paroxetina $20 \mathrm{mg}$ por dia e acompanhamento em um centro de saúde mental local. Teve alta com paroxetina e oxicodona $10 \mathrm{mg}$, 3 vezes ao dia.

Ao se apresentar à consulta nesta ocasião, a paciente está deitada de lado na maca de exame com os quadris flexionados. Reclama de dor abdominal espasmódica grave no abdome médio e inferior, com náusea. O exame novamente é negativo (Caixa 4), exceto pelo teste de Carnett positivo (ver legenda $\mathrm{n}^{\circ} 5$ da Figura). Solicita que seja hospitalizada para determinar a causa da dor e receber medicação intravenosa para seu alívio. Conclui-se pelo diagnóstico de síndrome da dor abdominal funcional (Caixa 8).

Paciente com dor abdominal constante ou frequentemente recorrente por pelo menos 6 meses:

7

• não associada com doença sistêmica conhecida - com perda da função diária inclusive de trabalho e socialização
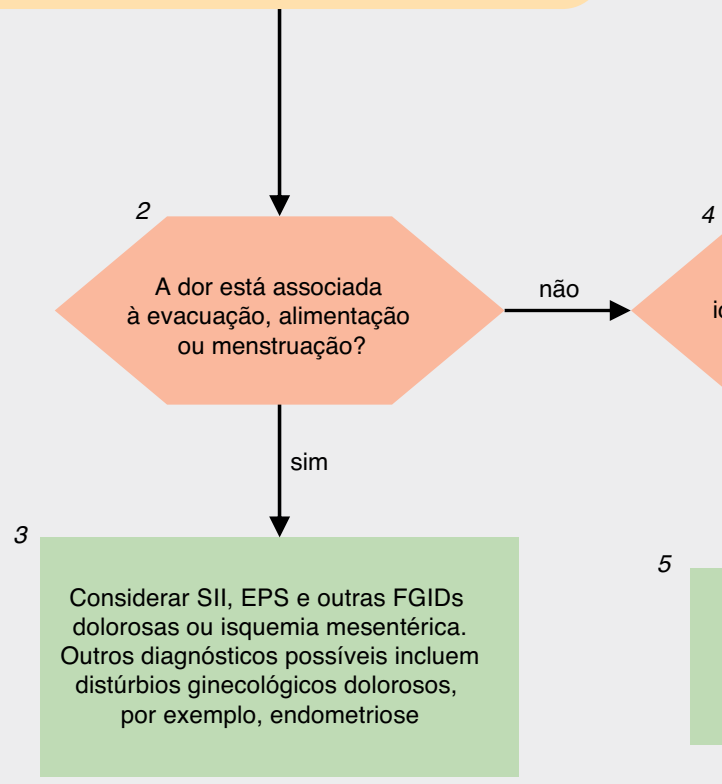

Realizar avaliação diagnóstica adequada para sinais de alarme ncaminhar ao profissional de saúde mental para excluir simulação de doença

FIGURA 1. Dor abdominal constante ou frequentemente recorrente

Legenda da figura 1

1. Dor abdominal constante ou frequentemente recorrente neste contexto se refere à dor que é constante, quase constante ou ao menos frequentemente recorrente, com dor ou desconforto ocorrendo todos os dias e quando a dor está presente há pelo menos 6 meses.

A dor está associada com algum comprometimento pessoal diário como absenteísmo no trabalho ou na escola e limitações nas atividades sociais. Sabe-se que não há doença sistêmica associada a esta dor. O histórico deve também incluir aspectos clínicos/psicossociais ${ }^{(1)}$. Durante a obtenção da história devem ser observados os comportamentos no relato dos sintomas ${ }^{(3)}$, que inclui a expressão verbal e não-verbal da variação de intensidade da dor, relato de sintomas intensos de forma urgente, minimização ou negação do papel de fatores psicossociais, solicitação de estudos diagnósticos adicionais, concentração da atenção na recuperação completa, busca frequente de assistência médica, atitude de assumir responsabilidade pessoal 
limitada para o autocontrole de sua condição e a solicitação de analgésicos narcóticos. Apesar das comunicações comportamentais não serem critérios para o diagnóstico, elas são aspecto geralmente observado neste contexto.

2. A história deve buscar características específicas da dor, especialmente associações com evacuações e alimentação, bem como qualquer relação com o ciclo menstrual.

3. Se a dor estiver associada às evacuações e resultar em diarreias frequentes ou em fezes duras infrequentes com alívio após a defecação (qualquer combinação de duas situações), deve então ser considerado o diagnóstico de síndrome do intestino irritável (SII) ${ }^{(1)}$ (ver o algoritmo 'dor abdominal recorrente ou crônica/desconforto com o hábito intestinal alterado'). Se a dor estiver localizada no epigástrio ou no quadrante superior direito, for grave, interromper as atividades diárias e for recorrente em intervalos diferentes (ou seja, não diariamente), deve ser considerado o diagnóstico de distúrbio funcional da vesícula biliar ou esfíncter de Oddi ${ }^{(13)}$ (ver os algoritmos 'dor recorrente similar à biliar' e 'dor similar à biliar pós-colecistectomia'). Se estiver localizada no epigástrio, for intermitente e não atender os critérios para distúrbio de vesícula biliar ou esfíncter de Oddi, deve ser considerada a dispepsia funcional (especificamente a síndrome da dor epigástrica [SDE] $)^{13}$ (ver o algoritmo 'dispepsia recorrente'). Deve ser considerado o diagnóstico de isquemia mesentérica crônica. Aspectos característicos podem incluir a dor exacerbada ao comer (angina intestinal) e a dor fora de proporções ao exame físico. Esta é uma determinação difícil de fazer, mas deve ser considerada no contexto de início recente do sintoma em paciente mais idoso, com histórico de doença vascular, juntamente com sintomas de náusea, vômitos e perda de peso. Diarreia também pode estar presente. A dor abdominal superior pode refletir envolvimento do tronco celíaco. Se estiver associada à menstruação ou por ela for agravada, pode indicar condições como a endometriose, sangramento uterino disfuncional ou outra doença ginecológica, que devem ser avaliadas através de exame pélvico, ultrassom pélvico e/ou pelo encaminhamento ao ginecologista. Exame pélvico irá auxiliar nesta determinação, examinando primeiro as estruturas pélvicas intravaginalmente (toque vaginal), complementando a seguir com o exame bimanual. Sugere-se a avaliação por um ginecologista, quando houver dúvida.

4. Deve ser realizado um exame físico completo. A elaboração do diagnóstico mínimo inclui hemograma, VSG/CRP, painel bioquímico, sangue oculto nas fezes (acima de 50 anos de idade). Os aspectos de alarme podem incluir achados anormais no exame físico, perda de peso não intencional, histórico familiar de câncer abdominal e anormalidades laboratoriais como anemia, hipoalbuminemia, prova de função hepática anormal, VSG elevado e sangue oculto positivo nas fezes.

5. Se os aspectos de alarme forem identificados pelo histórico, pelo exame físico ou pelos exames laboratoriais de triagem, devem ser consideradas outras fontes de dor abdominal. Um nível adequado de suspeita também pode ser necessário para diferenciar a dor crônica da parede abdominal da dor de origem visceral. A dor normalmente é localizada e aumenta com a contração dos músculos abdominais. O sinal de Carnett, em que a dor ou a sensibilidade aumenta com a tensão intencional dos músculos abdominais, pode ser obtido no exame físico nestes casos ${ }^{(14)}$. Apesar do sinal de Carnett positivo poder indicar dor da parede abdominal como a origem dos sintomas do paciente e, se obtido, poder ser levado em consideração, faz mais sentido, no contexto da história e do curso clínico do caso presente, que a dor da paciente esteja relacionada à hipersensibilidade central com hipervigilância. Portanto, no contexto do caso atual, um sinal de Carnett positivo seria considerado como a negação da dor visceral como a origem da dor e daria suporte à hipersensibilidade central mais do que à dor com origem na parede abdominal. Na realidade, o sinal de Carnett original, nesta aplicação, está sendo modificado para diferenciar os mecanismos de dor visceral e central.

6. A dor simulada ou a simulação de doença para se eximir de responsabilidade dizem respeito à produção intencional de sintomas físicos (ou psicológicos) falsos ou grosseiramente exagerados, motivados por incentivos externos. Isto pode estar relacionado a esquivar-se do trabalho, obter compensação financeira ou obter medicamentos. O DSM-IV indica que a simulação de doença para se eximir de responsabilidade deve ser altamente suspeita se for observada qualquer combinação do seguinte ${ }^{(15)}$ :

a. contexto médico-legal da apresentação (ou seja, encaminhado ao médico por um advogado para exame);

b. acentuada discrepância entre o estresse alegado pela pessoa, os sintomas ou a incapacidade e os achados objetivos;

c. falta de cooperação durante a avaliação diagnóstica e na aderência aos regimes de tratamento prescritos;

d. a presença de Transtorno da Personalidade Antissocial. Com base nos critérios do DSM-IV, três ou mais dos seguintes itens são exigidos para este diagnóstico: padrão predominante de descaso e de violação dos direitos dos outros conforme indicado por: I) comportamentos ilegais; II) desonestidade e mentira; III) impulsividade ou falta de planejamento antecipado; IV) irritabilidade e agressividade com lutas físicas ou assaltos; V) descaso negligente pela segurança do próprio e de outros; VI) irresponsabilidade com incapacidade de sustentar comportamento consistente no trabalho ou honrar compromissos financeiros; VII) ausência de remorso por ser indiferente ao ferir, maltratar ou roubar alguém.

A dor ou a doença simulada, para se eximir de responsabilidade, difere do distúrbio factício, pois que a motivação para a produção do sintoma na doença simulada é um incentivo externo, enquanto no distúrbio factício os incentivos externos estão ausentes. Ela difere do distúrbio de conversão ou do distúrbio somatoforme, pois os sintomas são produzidos intencionalmente e, portanto, ao contrário destas condições, o alívio frequentemente não é obtido por sugestão ou hipnose.

7. Sabe-se que a dor simulada não é fácil de ser detectada, portanto pode ser apropriado o encaminhamento a profissional de saúde mental para a confirmação esta suspeita. Ela não deve ser presumida, a menos que haja clara evidência da sua presença.

8. Na ausência de aspectos de alarme ou de anormalidades na avaliação e na presença de sintomas estáveis e duradouros, o diagnóstico da síndrome da dor abdominal funcional (SDAF) é altamente provável, se todos os critérios para este diagnóstico tiverem sido atendidos.

Os critérios diagnósticos de Roma III para SDAF são:

1. dor abdominal contínua ou quase contínua;

2. nenhuma relação ou apenas ocasional da dor com eventos fisiológicos (por exemplo, alimentação, defecação ou menstruação);

3. alguma perda do funcionamento diário;

4. a dor não é simulada;

5. sintomas insuficientes para atender os critérios de outro distúrbio gastrointestinal funcional que explicasse a dor, e,

6. critérios atendidos por pelo menos 3 meses com o início do sintoma pelo menos 6 meses antes do diagnóstico.

Se estes critérios forem atendidos, a reconfirmação com relação ao diagnóstico de SDAF e ao tratamento sintomático deve ser acompanhada por reavaliação dentro de 3 a 6 semanas ${ }^{(16)}$

Os fatores psicossociais, incluindo a depressão maior, o distúrbio de ansiedade, o distúrbio somatoforme, o enfrentamento mal adaptativo e o estresse da vida, incluindo abuso físico, sexual ou emocional, são comuns em pacientes com SDAF. Estes distúrbios podem ser identificados através da história psicossocial ou em alguns casos através de testes psicológicos ${ }^{(17)}$. O "Questionário Roma III de Alarme Psicossocial para Distúrbios Gastrintestinais Funcionais" (Apêndice A), que identifica "sinais de alarme" ou marcadores mais sérios de distúrbio psicossocial que indicariam o encaminhamento ao psiquiatra ou psicólogo, é ferramenta útil para a prática clínica. Estas questões são discutidas em detalhes por Creed et al. ${ }^{(18)}$, incluindo um algoritmo de tratamento com alertas para consulta com profissional de saúde mental. 
Outras opções de tratamento incluem terapia com ATC ou ISRS, terapia não farmacológica como a terapia cognitivo-comportamental (CBT), hipnoterapia ou psicoterapia dinâmica ou interpessoal ${ }^{(19)}$. Algoritmo para orientar as decisões de tratamento para SDAF é apresentado em Clouse et al. ${ }^{(3)}$. Em pacientes com acentuada incapacidade, uso errado de opióides e/ou problemas de dores múltiplas e difusas além da SDAF, nos quais estas estratégias de tratamento propiciam pouca ou nenhuma melhora, pode ser considerado o encaminhamento a um programa multidisciplinar especializado em dor crônica. Outra questão urgente a ser tratada que ocorre em pacientes com SDAF é o insensato uso de agentes narcóticos que pode resultar no desenvolvimento de síndrome do intestino narcótico ${ }^{(20)}$. Esta condição é caracterizada pelo uso antecipado de medicações narcóticas para alívio da dor, mas que paradoxalmente está associado com hiperalgesia. O tratamento exige a desintoxicação dos narcóticos e a substituição dos tratamentos, conforme observado acima.

\section{CONFLITO DE INTERESSE}

\section{Garantia do artigo: Rome Foundation.}

Contribuições específicas dos autores: Ami D. Sperber e Douglas A. Drossman são coautores e colaboradores da sessão SDAF e participaram de todos os aspectos desde o conceito inicial, dos rascunhos e da versão final.

Apoio financeiro: Financiado com subvenção da Rome Foundation.

Potenciais conflitos de interesse: Nenhum.

\section{REFERÊNCIAS}

1. Drossman DA. Functional abdominal pain syndrome. Clin Gastroenterol Hepatol. 2004;2:353-65.

2. Drossman DA. Presidential address: gastrointestinal illness and the biopsychosocial model. Psychosom Med. 1998;60:258-67.

3. Clouse RE, Mayer EA, Aziz Q, et al. Functional abdominal pain syndrome. In Drossman DA, Corazziari E, Delvaux M, et al. (eds). Rome III. The Functional Gastrointestinal Disorders, 3rd edn McLean, Virginia: Degnon Associates, 2006.

4. Maxton DG, Whorwell PJ. Use of medical resources and attitudes to health care of patients with "chronic abdominal pain". Br J Med Econ. 1992;2:75-9.

5. Dorn SD, Palsson OS, Thiwan SI, et al. Increased colonic pain sensitivity in irritable bowel syndrome is the result of an increased tendency to report pain rather than increased neurosensory sensitivity. Gut. 2007;56:1202-9.

6. Ringel Y, Drossman DA, Leserman JL, et al. Effect of abuse history on pain reports and brain responses to aversive visceral stimulation: an FMRI study. Gastroenterology. 2008;134:396-404.

7. Jones MP, Dilley JB, Drossman D, et al. Brain-gut connections in functional GI disorders: anatomic and physiologic relationships. Neurogastroenterol Motil. 2006;18:91-103.
8. Drossman DA. Brain imaging and its implications for studying centrally targeted treatments in irritable bowel syndrome: a primer for gastroenterologists. Gut.2005;54:569-73.

9. Drossman DA, Ringel Y, Vogt BA, et al. Alterations of brain activity associated with resolution of emotional distress and pain in a case of severe irritable bowel syndrome. Gastroenterology. 2003;124:754-61.

10. Grover M, Drossman DA. Psychopharmacologic and behavioral treatments for functional gastrointestinal disorders. Gastrointest Endosc Clin N Am. 2009;19:151-70.

11. Longstreth GF, Thompson WG, Chey WD, et al. Functional bowel disorders. Gastroenterology. 2006;130:1480-91.

12. Behar J, Carazziari E, Guelrud M, et al. Functional gallbladder and sphincter of Oddi disorders. Gastroenterology. 2006;130:1498-509.

13. Tack J, Talley NJ, Camilleri M, et al. Functional gastroduodenal disorders. Gastroenterology. 2006;130:1466-79.

14. Carnett JB. Intercostal neuralgia as a cause of abdominal pain and tenderness. Surg Gynecol Obstet. 1926;42:625.

15. First HB, Frances A, Pincus HA. DSM-IV-TR Guidebook. Washington, DC: American Psychiatric Publishing, 2004.

16. Drossman DA, Camilleri M, Mayer EA, et al. AGA technical review on irritable bowel syndrome. Gastroenterology. 2002;123:2108-31.

17. Levy RL, Olden KW, Naliboff BD, et al. Psychosocial aspects of the functional gastrointestinal disorders. Gastroenterology. 2006;130:1447-58.

18. Creed F, Levy RL, Bradley LA, et al. Psychosocial aspects of the functional gastrointestinal disorders. In Drossman DA, Corazziari E, Delvaux M, et al. (eds). The Functional Gastrointestinal Disorder., 3rd edn McLean, Virginia: Degnon Associates, 2006.

19. Drossman DA. Severe and refractory chronic abdominal pain: treatment strategies Clin Gastroenterol Hepatol. 2008;6:978-82.

20. Grunkemeier DM, Cassara JE, Dalton CB, et al. The narcotic bowel syndrome: clinical features, pathophysiology, and management. Clin Gastroenterol Hepatol. 2007;5:1126-39. 